\title{
Vanishing (Disappearing) Bone Disease in children - A review.
}

\author{
Nagaveni N.B. ${ }^{1}$, Radhika N.B. ${ }^{2}$, Umashankara K.V. ${ }^{3}$, Satisha T.S. ${ }^{4}$ \\ ${ }^{1}$ B.D.S., M.D.S., Reader, Department of Pedodontics \& Preventive dentistry Hitkarini Dental College \& Hospital, Jabalpur, \\ Madhya Pradesh, India \\ ${ }^{2}$ B.D.S., M.D.S., Senior Lecturer, Department of Orthodontics \& Dentofacial Orthopedics, School of Dentistry, Krishna Institute \\ of Medical Sciences, Karad, Maharashtra, India. \\ ${ }^{3}$ B.D.S., M.D.S., Professor, Department of Oral \& Maxillofacial surgery Hitkarini Dental College \& Hospital, Jabalpur, Madhya \\ Pradesh, India \\ ${ }^{4}$ B.D.S., M.D.S., Resident, Periodontics, Armed Forces Medical College Pune, Maharashtra, India
}

Correspondence:

Department of Pedodontics \& Preventive dentistry

Hitkarini Dental College \& Hospital,

Jabalpur, Madhya Pradesh, India

E-mail: nagavenianurag@gmail.com

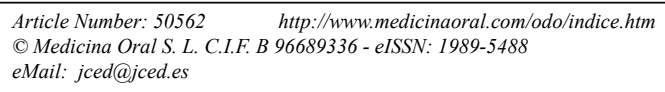

\begin{abstract}
Vanishing bone disease is a very rare, peculiar destructive condition of the skeletal system resulting in spontaneous and progressive resorption and disappearance of osseous structures with replacement by vascular, fibrous connective tissue. Children and adolescents are most commonly affected, although patients ranging in age from 18 months to 40 years have been reported. Its clinical presentation is highly variable largely depending upon the site of skeletal involvement. The maxillofacial territory and upper extremity bones are the predominant sites affected by this ailment. The etio-pathogenesis of this condition still remains poorly understood, the natural history and prognosis is unpredictable. Radiographic findings associated with vanishing bone disorder are particularly dramatic and characterized by complete resorption and disappearance of the affected bone leading to the definition of "vanishing bone" or "disappearing bone disease." Although various treatment options have been suggested, in general, no single treatment modality has proven effective in arresting this rare clinical entity. The present article provides an insight into the in-depth comprehensive review of literature pertaining to this fascinating musculoskeletal disorder in children.
\end{abstract}

Key words: Bone disorder, children, disappearing bone, Gorham's disease, radiation therapy, vanishing bone tumor. 


\section{Introduction}

"Vanishing bone disease" is an extremely rare idiopathic disease entity affecting the musculoskeletal system and characterized by extensive loss of bony matrix which is replaced by proliferating thin-walled vascular channels and fibrous tissue $(1,2)$. The radiographic features associated with this condition are particularly dramatic because in most of the cases, a complete resorption and disappearance of the affected bone occurred, and hence the terms "vanishing bone disease" or "disappearing bone disease" or "phantom bone" has been applied $(1,3)$. Most cases of vanishing bone disorder occurred in children or in adults less than 40 years of age (4-7). However, the disease has been described in patients as young as one month to as old as 75 years $(4,5)$. The bones of the maxillofacial region and upper extremity are the predominant osseous locations of this disease (6). In decreasing order of frequency it affects the mandible, scapula, proximal end of humerus, femur, rib, iliac bone, ischium and sacrum, although no area of the skeleton is immune. Mandible is the most common site of osteolysis in maxillofacial territory (6-8).

Still the precise etiology of this disorder remains poorly understood. There is no evidence of a malignant, neuropathic or infectious component involved in the causation of this disease and mechanism behind bone destruction or resorption is unclear. This rare disorder is not associated with gender or racial predilection too. However, approximately $60 \%$ of all cases with vanishing bone disease occurred in men (9). A variety of imaging methods have been used in evaluating patients suspected of having vanishing bone disease such as plain radiographs, radioisotope scans, computed tomography (CT), scintigraphy and magnetic resonance imaging (MRI) (6-13). But there is no consensus to date regarding the definite treatment for this condition. The suggested treatment strategies include surgical intervention, chemotherapy and radiation therapy. However, in general, no single treatment modality has proven effective in arresting the disease (10).

All previously reported cases involving the maxillofacial region have affected the mandible alone or with the maxilla or maxilla alone $(3,4,8,11-15)$. The clinical presentation is variable, and in the jaws, pain, tooth mobility, pathological fracture, facial deformity and malocclusion are early signs encountered (13-15). When vanishing bone disease involves the jaws of children, the role of pediatric dentist is extremely important in diagnosing promptly the disorder and preventing the functional and aesthetic consequences of advanced and extensive bone loss. Furthermore, mandibular involvement is considered a potential high-risk disease location, as severe progression from mandible to maxilla, skull and spine has been reported, thus leading to poor prognosis (1113). Accordingly, an early diagnosis of vanishing bone disease of the maxillofacial region is crucial to prevent patient's morbidity and mortality, and seems to be feasible because of the presence of early signs such as teeth mobility and bone loss.

The prognosis of vanishing bone disease is highly variable and unpredictable, ranging from minimal disability to death, because of the involvement of vital structures (16). Even if the osteolytic process often arrests spontaneously, the involvement of vital structures, such as the vertebral column and rib cage, is able to cause death $(1,2,6)$. The course of the disease is particularly insidious, as the osteolytic process is typically painless, allowing normal patient activity to continue while bone destruction occurs, making the patients susceptible to pathologic fractures (16).

Numerous articles on vanishing bone disease with different synonyms are published including both medical and dental disciplines like orthopaedic surgery, medicine, general surgery, otolaryngorhinology, neurosurgery, plastic surgery and maxillo-facial surgery (1-16). On comprehensive review of dental literature, it is evident that an overview of this rare disorder of the musculoskeletal system in pediatric field is not available. Each child suffering from vanishing bone disorder is unique and clinical presentation, and treatment varies individually, so the role of pediatric dentist is of utmost important in diagnosing the disorder and for early referral to receive comprehensive treatment and thereby preventing the complications. The aim of this review article is to make the pediatric dental fraternity aware of this rare mysterious disorder and discuss the pathogenesis, clinical symptoms, investigations, diagnosis, differential diagnoses and various treatment options for children suffering from vanishing bone disease.

\section{Review of literature}

Synonyms

Numerous names have been used in the world literature to describe this rare disorder such as Gorham's disease, Gorham-Stout syndrome, phantom bone disease, massive osteolysis, disappearing bone disease, acute spontaneous absorption of bone, hemangiomatosis, essential osteolysis and lymphangiomatosis, Gorham's vanishing bone disease, thoracic lymphangiomatosis, vanishing bone tumor, Gorham's syndrome, idiopathic massive osteolysis, massive Gorham osteolysis, Morbus GorhamStout disease, progressive massive osteolysis, disseminated lymphangiomatosis, Hajdu-Cheney syndrome, idiopathic multicentric osteolysis, Acro-osteolysis syndrome, lymphangiomatosis-hepatic disease, lymphangiomatosis-splenic disease, cystic angiomatosis of bone, and diffuse pulmonary lymphangiomatosis (6-20).

Historical perspective

Idiopathic osteolysis was first described in 1838 (16) and with term "vanishing bone disease" was first des- 
cribed by J.B.S. Jackson in 1872 in a 12 -year-old boy having complete osteolysis of the humerus (20). The clinical features were so impressive that which provoked the author to describe the case as the "Boneless arm" (16) Since then approximately 200 cases of vanishing bone disease affecting various skeletons has been reported in the literature. In 1954, Gorham and colleagues (21) presented two patients affected by this condition. Of the two cases, one was a 16-year-old boy with right clavicle and scapula involvement. This patient eventually developed chylothorax, a serious complication of vanishing bone disease and finally expired. The other patient was 44-years-old male, who also had involvement of the right clavicle and scapula. In addition to these two cases, they reported a review of 16 cases with this condition. Later in 1955, Gorham and Stout ${ }^{1}$ published the first overview of vanishing bone disorder and reported 24 additional cases. They further characterized the main pathologic features of this disease as non-malignant intraosseous proliferation of vascular tissue that caused massive osteolyis. Hence vanishing bone disease is also well known as "Gorham's disease."

Vanishing bone disease during pregnancy has been reported in 1993 by Porter et al (22). The infant was delivered by forceps, with a good outcome. Two other cases of this condition with natural pregnancies were also presented by Kulenkampff et al (23).

\section{Etiopathogenesis and pathophysiology}

The exact etiology and the mechanism behind bone destruction and resorption in vanishing bone disease is still unknown. There is no genetic transmission and the incidence of the disease may be linked to a history of minor trauma $(16,20)$.

The pathological process involves the replacement of normal bone by an aggressively expanding but nonneoplastic vascular tissue $(24,25)$. It is unknown what stimulus sets off this change in bone and also whether all bones of the affected individual are equally likely to react in this adverse fashion. Since it is most commonly seen with trauma $(16,20)$, it is reasonable to deduce that the traumatic episode initiates the sequence of events. Since neovascularisation of the blood clot that forms in between fragments of a fracture is the first step in bone healing it is tempting to speculate that some error in this process, or in the control of this process, is the root cause of vanishing bone disease.

Campbell (26) hypothesized that post-traumatic arterial hyperemia was responsible for bone resorption, but this was rejected by Gorham et al (21). The same author postulated an angioma might act as a shunt, thereby increasing local oxygen tension (21). In most cases, trauma was relatively trivial, and in some cases, trauma did not occur (6). As with many other diseases, the role of trauma in vanishing bone disease may be to signal the presence of a preexisting abnormality. Whereas Knoch (27) suggested that a previous silent hamartoma can become active after a minor trauma, leading to bone resorption. Heyden and colleagues (28) found strong activity of both acid phosphatase and leucine aminopeptidase in mononuclear periavascular cells that were in contact with remaining bone, perhaps indicating that these cells are important in the process of osseous resorption.

According to Thompson and Schurman (29), the disease is a primary aberration of vascular tissue in bone, related to hyperemic granulation tissue. Young et al (30) stated endothelial dysplasia of blood and lymphatic vessels could lead to osteolysis. Osteoclasts are the only cells capable of resorbing bone. Therefore, it is presumed that vanishing bone disease may represent a pathologic derangement of osteoclastic activity. Any defect of the osteoclasts could lead to idiopathic osteolysis. It has also been suggested that thyroid c cells and calcitonin may play an important role in the pathogenesis of this condition (31).

Clinical presentation and symptoms

Clinical onset of vanishing bone disease has been reported to occur anytime from childhood to adulthood and has been noted to be posttraumatic in many cases $(4-7,32)$. It usually manifests unilaterally and includes focal lymphatic proliferation involving one bone or contiguous bones such as vertebrae. Adjacent soft tissue can also be involved, producing diffuse muscle atrophy. Any part of the skeleton can be affected and the process may affect the appendicular or the axial skeleton (33). The shoulder and the maxillofacial region are the most common sites of involvement $(6-8,11,12,33)$; however, various locations such as the humerus, scapula, clavicle, ribs, sternum, pelvis and femur can be affected by vanishing bone disease. The disorder is also known to occur at other sites such as the skull, spine, hand and foot (34). If the shoulder girdle or thoracic cage is involved, then $17 \%$ of patients develop chylothorax which leads to hypoproteinemia, malnutrition and immunosuppression; this is often fatal with a mortality rate of $64 \%(35,36)$. Clinical manifestations of vanishing bone disease may vary and depend on the affected site (6). Dull aching, swelling, weakness in the affected extremity, and skeletal deformities are prominent symptoms, whereas, some patients presented with a history of insidious onset of pain, limitation of motion, and progressive weakness in the involved limb. The usual presenting symptom is localized pain usually secondary to fracture. However, deformity of bone may be the only complaint as reported in some cases $(6-8,11-15,36)$.

Vanishing bone disease in the maxillofacial territory Among the 200 vanishing bone disorder cases reported in the international literature, approximately 50 cases have been found to affect bones of the maxilla-facial region (usually the mandible) $(6-8,11,12)$. The first case reported in the jaws was by Romer (37) in 1924, in a 
31-year-old woman. Frederiksen et al (13) observed that the mandible is involved most frequently, followed by the maxilla with varying degrees of involvement of contiguous structures including the hard palate, sphenoid bone, and zygomatic bone.

Initial presentation of vanishing bone disorder with maxillofacial involvement may be evident as mobile but vital teeth with accompanying gingival hemorrhage. Hypoplasia, pain, malocclusion, and resorption of the affected alveolar and adjacent bone are other features $(6-8,11,12,38-40)$. Involvement of the temporomandibular joint by vanishing bone disease can be mistaken for temporomandibular dysfunction and mandibular involvement may present as pathological fracture (41). Maxillofacial involvement, seen first with pain, malocclusion and deformity, is reported in $30 \%$ of cases (42). Jaw bone involvement can also affect mastication, swallowing, speaking, and respiratory function, as well as cosmetic appearance of the affected child.

Investigations and diagnosis

The diagnosis of vanishing bone disease is based on thorough clinical examination, radiologic imaging studies, and histopathologic examination of the affected bone $(6,13,24,33)$. The standard laboratory findings are not specific and of no value in diagnosis of this condition (24). An elevated serum alkaline phosphatase level has been reported in some patients with an associated fracture (6). A variety of imaging methods such as plain radiographs, radioisotope scans, computed tomography and magnetic resonance imaging (32) have been used in evaluation of patients with vanishing bone disorder $(24,31,43)$.

Devlin et al (25) also showed increased levels of interleukin-6 might act as a potential mediator of increased osteoclast activity in patients with vanishing bone disease. Assoun et al (44) used computed tomography to differentiate soft-tissue involvement and the extent of bone osteolysis and also to facilitate biopsy of the affected bone. Radioisotope bone scan may demonstrate increased vascularity on initial images and subsequently, an area of decreased uptake corresponding to the site of diminished or absent osseous tissue $(24,43)$. However, these results have been found variable. Conventional angiography could not reveal the pathologic vascular changes according to Szabo and Habre (45), and scintigraphy (43) is not reliable because of variable accumulation of isotopes at the lesion site. MRI findings of this condition are non-specific and variable. T1 weighted spin echo MRI shows uniform low signal intensity in involved bones, whereas increased signal intensity shows in $\mathrm{T} 2$ weighted spin echo images $(43,44)$.

Radiographic findings

The most dramatic feature of vanishing bone disorder is its radiographic appearance $(1-3,13,43)$. Naden (46) in 1995, suggested radiographs are the best tools for de- tecting vanishing bone disease. Radiographic findings in patients with this disease were well described by Resnick (47).

During initial stage of the lesion radiolucent foci appear in the intramedullary or subcortical regions, resembling findings seen in patchy osteoporosis. Subsequently, slowly progressive atrophy, dissolution, fracture, fragmentation and disappearance of a portion of the bone occurs with tapering or pointing of the remaining osseous tissue and atrophy of soft tissues. The disease process can extend to contiguous bones; the intervening joints afford no protection to extension of the disease $(1-3,43)$.

Patrick in 1976 (48) classified radiological changes of vanishing bone disease into four stages as:

Radiolucent foci, resembling patchy osteoporosis

Shrinkage of shaft of bones by tapering of the ends ("Sucked candy" appearance)

Complete resorption of the bone unless there is spontaneous arrest and Progression to adjacent bones and joints

Histo-pathological features

Histo-pathological examination of tissue from an affected area in vanishing bone disease reveals a non-specific vascular proliferation with fibrous connective tissue, sometimes containing lymphocytes and plasma cells $(13,24)$. The vascular proliferation consists of thinwalled, often dilated, vascular or lymphatic channels of varying sizes (6). Angiomatosis, however, is not always a histopathological feature and has been questioned in the literature as an intrinsic characteristic.

Histological diagnosis depends on the phase in which the disease is diagnosed. Heffez et al (49) in 1983, described 2 phases of this disease. In the first phase, increased vascular concentration in the bone-displacing fibrous tissue part is seen; and the second phase represents only fibrous tissue. The presence and number of osteoclasts vary significantly in vanishing bone disease. In most reported cases the osteoclastic activity was minimal or nonexistent, whereas in other cases, osteoclasts were easily identifiable. If present, osteoclastic activity is seen in the interface between the vascular channels and the cortex. Heffez et al (49) suggested eight criteria (histopathologic and clinical) for definitive diagnosis of vanishing bone disease which help distinguish from other destructive bone disorders as follows:

1. Positive biopsy in terms of angiomatous tissue presence

2. Absence of cellular atypia

3. Minimal or no osteoclastic response and absence of dystrophic calcification

4. Evidence of local bone progressive resorption

5. Non-expansive, non-ulcerative lesion

6. Absence of visceral involvement

7. Osteolytic radiographic pattern and

8. Negative hereditary, metabolic, neoplastic, immu- 
nologic and infectious etiology

\section{Differential diagnosis}

For differential diagnosis of vanishing bone disease in chidlren, other causes of osteolysis should be considered such as hereditary osteolysis, skeletal angiomas, essential osteolysis, trauma (Sudeck atrophy), infection, endocrine diseases, various nervous system diseases, and malignancies (primary or secondary) $(6,47,50)$. In all of the other disorders, the histologic and clinical features are distinct from vanishing bone disease. Essential osteolysis causes resorption of carpal and tarsal bones with progressive renal failure but without vascular proliferation (33). Skeletal angiomas have less growth potential and tend to preserve bony cortex without spreading into adjacent bone. In hereditary osteolysis the disease process starts in childhood, tends to be multicentric, and involves primarily the hands and foot (carpotarsal osteolysis) but vascular proliferation is not seen. Malignant disease must be ruled out histologically, although lack of soft tissue mass points away from the presence of a neoplasm. Sclerosis and periosteal bone formation, seen radiographically in osteomyelities, are not features of vanishing bone disease. There are no serum abnormalities or generalized osseous changes as with metabolic conditions $(33,37,47)$.

Therefore, it is worth emphasizing that for all children who present with skeletal osteolysis, a thorough history and meticulous physical examination should be undertaken first. Appropriate blood test and radiographic studies should be made mandatory to rule out other common underlying causes of osteolysis in children.

\section{Management}

Extensive review of current literature showes that there is no universally accepted, most efficacious treatment for vanishing bone disease. The goal of the treatment is to arrest progression of the osteolysis as remineralization or reformation of affected bone is not seen. Therapeutic treatment with estrogens, magnesium, calcium, vitamin $\mathrm{D}$ and vitamin B12, fluoride, and calcitonin as well as cisplatin, actinomycin D, aluminium acetate solution, ultraviolet radiation, somatotrophin, amino acids, or even placental extracts or transfusions of placental blood have been tried and proved unsuccessful $(6,33)$. Anavi et al (11) suggested treatment for vanishing bone disorder should include surgery, radiotherapy and various medications, either alone or in combination.

Surgical intervention has been proposed as the method of choice by many authors that involve local resection of the affected bone and reconstruction using bone grafts and or prostheses (51-53). But bone grafting techniques have yielded poor results, and many authors observed a high incidence of bone graft resorption (52). Sympathectomy also has been suggested but with no effect on the disease (6). Immobilization of the involved extremity does not affect the prognosis (33). In children with spinal involvement, instability must be watched for and managed early. Spinal localizations are best treated by segmental fixation extended to normal vertebrae (6). Apart from standard orthopaedic treatment for fractures, non unions and deformities, the medical treatment for vanishing bone disease can be categorized into two groups; chemotherapy (anti-osteoclastic medication like bisphosphonates and alpha-2b-interferon) and radiation therapy $(6,33)$. Chemotherapy has been advocated to be successful in some cases $(6,33,54)$.

Radiotherapy acts by accelerating sclerosis of the proliferating blood vessels and prevents re-growth of these vessels. Results obtained with radiation therapy have been equivocal, although in few patients, apparent arrest has been produced. Radiation therapy, especially when used early in the course of the disease, appears to be the only accepted form of treatment with a greater chance of success. However, it is difficult to determine the potential of radiation therapy accurately as its use in the treatment of vanishing bone disease has been documented as only case reports $(6,33,55,56)$. It is reported that the use of total doses from 30 to $45 \mathrm{~Gy}$ are most effective. Fontanesi (55) showed excellent results using a total dose of $15 \mathrm{~Gy}$ in a case that involved the upper extremity. Hanly et al (56) reported rapid relief of symptoms and prevention of further osseous destruction during a 6-year followup period with a total dose of 3000 rads. Re-growth of bone after radiation therapy is unusual but has been reported in some patients $(26,57)$. Radiation therapy can be used for the management of chylothorax and pleural effusion in cases who may not be suitable candidates for an extensive surgical procedure due to their poor general health, and for those who have failed surgical treatment $(58,59)$. However, the disadvantages of radiation therapy including the possibility of acute side effects such as gastrointestinal tract irritation with resultant nausea and or vomiting, and radiation induced pneumonia should be considered. Other treatment options that have been used effectively for the management of chylothorax are pleurodesis, pleurectomy, thoracic duct ligation, oral clodronate, bleomycin and interferon alfa-2b therapy (48). Of course, the usefulness of all these treatments is also shaped by the severity of the disease (for example, surgery would be impractical in patients with widespread vertebral involvement) and the urgency of the patient's condition (36).

In children and adolescents when considering radiotherapy the complications like potential for secondary malignant transformation, growth restrictions and damage to tooth formation and eruption should be considered before embarking on this mode of treatment $(6,7,18)$.

Prognosis

The prognosis of vanishing bone disease varies from slight disability to death due to involvement of vital skeletal structures, malnutrition, lymphocytopenia or 
infection (6). It has been reported that more than $15 \%$ of patients die as a result of their disease. Severe disability occurs due to involvement of the pelvis, thorax, and cervical spine (52). Neurological complications increase the mortality of this condition to $33.3 \%$. However, the disease usually remains localized within a skeletal region and undergoes eventual spontaneous arrest. In patients complicated with chylothorax, the mortality rate is more than $50 \%(36,48,59)$. Of 22 reported cases of this condition related chylothorax between 1960 to 2000,12 patients survived and 10 were died. Of the 12 surviving patients, 8 were surgically managed and 4 were medically managed, all in a trial and error basis $(36,48,59)$.

New ray of hopes

Due to the rarity of the disease and non-availability of most efficacious treatment options, recently research has begun on genetic mechanisms in treating vanishing bone disease (6,33). A vascular endothelial growth factor receptor (VEGRr3) that is specific for lymphatics has been identified. Three genes associated with inherited lymphedema have also been discovered. Animal models simulating lymphedema are currently being studied. It is possible that the pathogenesis of lymphangiomatosis and vanishing bone disease is different; lymphatic malformations that arise during early childhood might stem from aberrant sequestrations of lymphatic vessels, while the acquired proliferation of small lymphatics observed in vanishing bone disease might be driven by lymphangiogenic growth factors.

\section{Complications}

Complications of vanishing bone disease may be neurological or pulmonary and they usually depend on the affected bones $(60,61)$. Chylothorax and hemothorax also may be associated with this condition $(48,60,61)$. Because there have been 25 reported cases of chylothorax as a complication of vanishing bone disease in that 10 cases were bilateral $(6,33,48)$. Chylothorax can be resulted from invasion of the thoracic duct by the lymphangioma or penetration of vascular dysplasia into the pleural cavity $(35,48)$. Paraplegia related to spinal cord involvement may occur in patients who have involvement of vertebrae with resultant osteolysis $(6,62)$. Thoracic cage, pulmonary, or pleural involvement can lead to compromise of respiratory function and death can ensue $(6,61,62)$. Infections of bone and septic shock, although rare, have also been reported (58).

\section{Discussion}

Vanishing bone disease is a combined clinical, radiographic and histologic entity characterized by benign vascular proliferation originating in bone and producing complete lysis of all or a portion of the bone. This condition can involve men or women and any age group although most cases are reported before the age of 40 years (4-7). There is no biochemical or endocrine abnormality asso- ciated with the condition. The skeletal structures affected include the thorax, hip bones, spine, maxilla and mandible although no area of the skeleton is immune. This condition does not occur often enough to be recognized early and take many years for the condition to be recognized even though it is quite well described (6-8, 11-13). In most cases, the condition is not recognized until a fracture occurs following trivial trauma and bone healing is delayed or subverted $(25,63)$. There are no reported cases of metastasis to date, although polyostotic and monostotic lesions have been described (64). In some cases, the disease has spontaneously arrested and in others progression of disease has resulted in death of the patient (6). Clinically the course of this disease is divided into two phases, an active phase of bone resorption and lysis and a second, generally dormant phase $(13,33)$. The succession of phases is unpredictable, taking a few months to many years. Chylothorax and pleural effusions may occur due to mediastinal extension of the disease process from the involved vertebra, scapula, rib or sternum and can be life threatening $(48,60,61)$. A high morbidity and mortality is seen in patients with spinal and or visceral involvement.

The etiology of this condition still remains unknown, although an initial trauma, or modification in local conditions such as variation of $\mathrm{PH}$, inflammations are suspected $(16,20,24,25)$. Histologically bone is replaced by abundance of thin-walled, capillary-sized vascular channels and at a later stage by fibrous connective tissue $(13,24)$. Diagnosis of vanishing bone disease is often delayed and most difficult in majority cases as laboratory findings are found within normal limits. So a high index of clinical suspicion together with characteristic radiographic and histopathological findings is more helpful for making an early diagnosis.

Imaging features of vanishing bone disease on conventional radiography, CT and MRI have been reported in the literature and most of the cases failed to show the vasculature entity of the lesion an important pathologic feature of the disease $(1-3,13,24,32,43)$. On radiographs, vanishing bone disease appears as unilateral, partial or total disappearance of contiguous bones, tapering of bony remnants, and absence of a sclerosing or osteoblastic reaction. Pertinent negative radiographic findings are absence of phleboliths, vascular or soft-tissue calcification, coarse trabeculation, and any related bony abnormality with the exception of demineralization resulting from disuse.

Due to the rarity of this disease entity there is no standard therapy available in controlling the condition, however, surgical treatment, anti-osteoclastic medication and radio therapy have been suggested.

\section{Conclusion}

"Vanishing bone disease" also known as "Disappearing 
bone disease" is an extremely rare bone disorder with no known exact etiopathogenesis. As the bones of the maxillofacial territory are frequently affected by this ailment and due to rarity of this condition it is important for pediatric dentist to be aware of its occurrence as a rare cause of osteolysis in the maxillofacial skeleton of children which subsequently causing functional, cosmetic impairment and if untreated leading to serious complications.

\section{References}

1. Gorham L, Stout A. Massive osteolyisis (acute spontaneous absorption of bone disappearing bone): its relation to hemangiomatosis. J Bone Joint Surg Am, 1955; 37: 985-1004

2. Choma, ND, Biscotti CV, Bauer TW, Mehta AC, Licata AA. Gorham's syndrome: a case report and review of the literature. Am J Med, 1981; 83: 1151-1156.

3. Tsang W, Tong A, Chow L, Ng I. Massive osteolysis (Gorham disease) of the maxillofacial skeleton. J Oral Maxillofac Surg, 2004; 62: $225-230$.

4. Motamedi MH, Homauni SM, Behnia H. Massive osteolysis of the mandible: a case report. J Oral Maxillofac Surg, 2003; 61: 957963.

5. Fisher KL, Pogrei MA. Gorham's syndrome (massive osteolysis): a case report. J Oral Maxillofac Surg 1990; 48: 1222-1225.

6. Patel DV. Gorham's disease or Massive osteolysis. Clin Med Res, 2005; 3(2): 65-74.

7. Ricalde P, Ord RA, Sun CC. Vanishing bone disease in a five year old: report of a case and review of the literature. Int J Oral Maxillofac Surg, 2003; 32(2): 222-62.

8. Moizan H, Talbi M, Devauchelle B. Massive mandibular osteolysis: a case with non-contributive histology. J Oral Maxillofac Surg, 2007; 65: 772-776.

9. Huvos AG. Bone tumors, diagnosis, treatment and prognosis. 2nd ed. Phildelphia, PA, WB Saunders, 1991.

10. Malik R, Malik R, Tandon S, Tandon P. Skeletal angiomatosis rare cause of bone destruction: A case report with review of literature. Indian J Pathol Microbiol, 2008;1(4): 515-518.

11. Anavi Y, Sabes WR, Mintz S. Gorham's disease affecting the maxillofacial skeleton. Head Neck, 1989; 11: 550-557.

12. Benhalima H, Lazrak A, Boulaich M, Mezahi M, Ourlai A, Kzadri $\mathrm{M}$, et al. Massive osteolysis of the maxillo-facial bones: case report and review of the literature. Odontostomatol Trop, 2001; 24: $35-40$.

13. Frederikson NL, Wesley RK, Sciubba JJ, Helfrick J. Massive osteolysis of the maxillofacial skeleton: a clinical, radiographic, histologic, and ultrastructural study. Oral Surg Oral Med Oral Pathol, 1983; 55: 470-480.

14. Phillips RM, Bush OB Jr, Hall HD. Massive osteolysis (phantom bone, disappearing bone): report of a case with mandibular involvement. Oral Surg Oral Med Oral Pathol, 1972; 34: 886-896.

15. Holroyd I, Dillon M, Roberts GJ. Gorham's disease: a case report (including dental presentation) of vanishing bone disease. Oral Surgery Oral Med Oral Pathol Oral Radiol Endod 2000; 89; 125 129.

16. Jackson JBS. A boneless arm. Boston Med Surg J, 2000; 18, 368369.

17. Aviv RI, McHugh K, Hunt J. Angiomatosis of bone and soft tissue: a spectrum of disease from disuse lymphangiomatosis to vanishing bone disease in young patients. Clin Radiol, 2001; 56(3): 184-90.

18. Prasanna R, Sankar J, Ramachandran P. Gorham's disease: Vanishing bone syndrome. Indian Pediatr, 2009; 46: 255-256.

19. Mignogna MD, Fedele S, Lo Russo L, Lanza A, Marenzi G, Sammartino G. Gorham's disease of the mandible mimicking periodontal disease on radiograph. J Clin Periodontol, 2005; 32(9): 1022-6.

20. Jackson JBS. Absorption of humerus after fracture. Boston Med
Surg J, 1897; 10: 245-247.

21. Gorham LW, Wright AW, Shultz HH, Maxon FC. Disappearing bones. A rare form of massive osteolysis: a report of two cases, one with autopsy findings. Am J Med, 1954; 17: 674-682.

22. Porter KB, O'Brien WF, Towsley G, Cates JD, Watts DB. Pregnancy complicated by Gorham disease. Obstet Gynecol. 1993; 81(5): 808-810.

23. Kulenkampff HA, Richter GM, Hasse WE, Adler CP. Massive pelvic osteolysis in the Gorham-Stout syndrome. Int Orthop, 1990; 14(4): 361-366.

24. Dickson GR, Hamilton A, Hayes D, Carr KE, Davis R, Mollan RA. An investigation of vanishing bone disease. Bone, 1990; 11: 205-210.

25. Devlin RD, Bone HG, Roodman GD. Interleukin-6: a potential mediator of the massive ostelolysis in patients with Gorham-Stout disease. J Clin Endocrinol Metab, 1996; 81: 1893-1897.

26. Campbell J, Almond HG, Johnson R. Massive osteolysis of the humerus with spontaneous recovery. J Bone Joint Surg Am, 1975; 57: 238-240.

27. Knoch HG. Die Gorhamsche Krankheit aus Klinischer Sicht. Zentralbil chir. 1963.

28. Heyden G, Kindblom LG, Nielsen JM. Disappearing bone disease: a clinical and histological study. J Bone Joint Surg Am, 1977; 59(1): 57-61.

29. Thompson JS, Schurman DJ. Massive osteolysis: case report and review of the literature. Clin Orthop Relat Res, 1974; 103: 206211.

30. Young JW, Galbraith M, Cunningham J, Roof BS, Vujic I, Gobien $\mathrm{RP}$, et al. Progressive vertebral collapse in diffuse angiomatosis. Metab Bone Dis Relat Res, 1983; 5(2): 53-60.

31. Korsic M, Jelasic D, Potocki K, Giljevic Z, Aganovic I. Massive osteolysis in a girl with agenesis of thyroid C cells. Skeletal Radiol, 1998; 27: 525-528.

32. Vinee P. Tanyu MO, Hauenstein KH, Sigmund G, Stover B, Adler CP. CT and MRI of Gorham syndrome. J Comput Assit Tomogr, 1994; 18(6): 985-989.

33. Papadakis BSA, Khaldi L, Babourda EC, Papadakis S, Mitsitsikas T, Sapkas G. Vanishing bone disease: review and case reports. Orthopedics, 2008; 31(3): 278.

34. Green HD, Mollica AJ, Karuza AS. Gorham's disease: a literature review and case reports. J Foot Ankle Surg, 1995; 34: 435-441.

35. Fujiu K, Kanno R, Suzuki H, Nakamura N, Gotoh M. Chylothorax associated with massive osteolysis (Gorham's syndrome). Ann Thorac Surg, 2002; 73: 1956-1957.

36. Duffy BM, Manon R, Patel RR, Welsh JS. A case of Gorham's disease with chylothorax treated curatively with radiation therapy. Clin Med Res, 2004; 3: 83-86.

37. Romer O. Die pathologie der Zahne. In: von Henke F, Lubarsch O (eds). Handbuch der speziellen pathologischen anatomie and histology. Vol 4, Berlin: Springer Verlag, pp 135-499, 1924.

38. Raghuveer HP, Jayaleshmy R. Gorham's massive osteolysis of the mandible - a progressive radiographic presentation. Dentomaxillofac Radiol, 2009; 38: 292-295.

39. Klein M, Metelmann, HR, Gross U. Massive osteolysis (GorhamStout syndrome) in the maxillofacial region: an unusual manifestation. Int J Oral Maxillofac Surg, 1996; 25: 376-3788.

40. Ohya T, Shibata S, Takeda Y. Massive osteolysis of the maxillofacial bones: report of two cases. Oral Surg Oral Med Oral Pathol, 1990; 70: 698-703.

41. Schiel H, Prien J. Seven-year follow-up of vanishing bone disease in a 14-year-old girl. Head Neck, 1993; 15: 352-356.

42. Bouloux, GF, Walker DM, McKellar G. Massive osteolysis of the mandible. Report of a case with multifocal bone loss. Oral Surg Oral Med Oral Pathol Oral Radiol Endod, 1999; 87: 37-361.

43. Spieth ME, Greenspan A, Forrester DM, Ansari AN, Kimura RL, Gleason-Jordan I. Gorham's disease of the radius: radiographic, scintigraphic, and MRI findings with pathologic correlation. A case report and review of the literature. Skeletal Radiol, 1997; 26: 659-663. 
44. Assoun J, Richardi G, Railhac JJ, Le Guennec P, Caulier M, Dromer C, et al. CT and MRI of massive osteolysis of Gorham. J Comput Assist Tomogr, 1994; 18(6): 981-984.

45. Szabo C, Habre W. Gorham syndrome: anesthetic management. Anaesthesia. 2000; 55(2): 157-159.

46. Naden BA. When bone disappears. RN, 1995; 58(10): 26-28.

47. Resnick D. Chapter 89. Osteolysis and chondromysis. In: Resnick $\mathrm{D}$, ed. Diagnosis of bone and joint disorders. Fourth edition. Philadelphia, Pennsylvania, USA: WB Saunders Company: Volume 5, pp 4920-4944, 2002.

48. Patrick JH. Massive osteolysis complicated by chylothorax successfully treated by pleurodesis. J Bone Joint Surg Am, 1976; 58: 347-349.

49. Heffez L, Doku, HC, Carter BL, Feeney JE. Perspectives of massive osteolysis: report of a case and review of the literature. Oral Surg Oral Med Oral Pathol, 1983; 55(4): 331-343.

50. Perschbacher SE, Perschbacher KA, Pharoah MJ, Bradley G, Lee L, Yu E. Gorham's disease of the maxilla: a case report. Dentomaxillofac Radiol, 2010; 39(2): 119-23.

51. Turra S, Gigante C, Scapinelli R. A 20-year follow-up study of a case of surgically treated massive osteolysis. Clin Orthop Relat Res, 1990; 250: 297-302.

52. Woodward HR, Chan DP, Lee J. Massive osteolysis of the cervical spine: a case report of bone graft failure. Spine, 1981; 6: 545-549.

53. Cannon SR. Massive osteolysis: a review of seven cases. J Bone Joint Surg Br, 1998; 68: 24-28.

54. Schnall SB, Vowels J, Schwinn CP, Wong D. Disappearing bone disease of the upper extremity. Orthop Rev, 1993; 22(6): 617-620.

55. Fontanesi J. Radiation therapy in the treatment of Gorham disease. J Pediatr Hematol Oncol, 2003; 5(10): 816-817.

56. Hanly JG, Walsh NM, Bresnihan B. Massive osteolysis in the hand and response to radiotherapy. J Rheumatol, 1985; 12(3): 580-582.

57. Bek V, Halcl Z, Kolar J, Bednar B. Radiotherapy of the GorhamStout syndrome. Cesk Radiol, 1981; 35: 291-298.

58. Kery L, Wouters HW. Massive osteolysis: report of two cases. J Bone Joint Surg, 1970; 52: 452-459.

59. McNeil KD, Fong KM, Walker QJ, Jessup P, Zimmerman PV. Gorham's syndrome: a usual fatal cause of pleural effusion treated successfully with radiotherapy. Thorax, 1996; 51: 1275-1276.

60. Gherlinzoni F, Rocco M. Progressive teleangiomatous osteolysis: case report. Itl J Orthop Traumatol, 1983; 9(4): 515-519.

61. Meller JL, Cure-Scott M, Dawson P, Besser AS, Shermeta DW. Massive osteolysis of the chest in children: an unusual cause of respiratory distress. J Pediatr Surg, 1993; 28(12): 1539-1542.

62. Halliday DR, Dahlin DC, Pugh DG, Young HH. Massive osteolysis and angiomatosis. Radiology,1964; 82: 637-644.

63. Lee S, Finn L, Sze RW, Perkins JA, Sie KC. Gorham Stout syndrome (disappearing bone disease): two additional case reports and a review of the literature. Arch Otolaryngol Head Neck Surg, 2003; 129: 1340-1343.

64. Vigorita VJ, Magitsky S, Bryk E. Gorham's disease: an autopsy report. Clin Orthop Relat Res, 2006; 451: 267-273. 